\title{
Soter e soteria dal V secolo alle soglie dell'età ellenistica: qualche considerazione
}

Soter and soteria from the Fifth Century B.C. to the Threshold of the Hellenistic Age: Some Considerations

\section{Giorgio Camassa}

\section{(2) OpenEdition}

\section{Journals}

\section{Edizione digitale}

URL: https://journals.openedition.org/mythos/1723

DOI: $10.4000 /$ mythos. 1723

ISSN: 2037-7746

\section{Editore}

Salvatore Sciascia Editore

Notizia bibliografica digitale

Giorgio Camassa, «Soter e soteria dal V secolo alle soglie dell'età ellenistica: qualche considerazione», Mythos [Online], 14 | 2020, online dal 31 décembre 2020, consultato il 05 octobre 2021. URL: http:// journals.openedition.org/mythos/1723 ; DOl: https://doi.org/10.4000/mythos.1723

Questo documento è stato generato automaticamente il 5 octobre 2021.

Mythos 


\section{Soter e soteria dal V secolo alle soglie dell'età ellenistica: qualche considerazione}

Soter and soteria from the Fifth Century B.C. to the Threshold of the Hellenistic Age: Some Considerations

Giorgio Camassa

per Elisabetta

1 Soter e soteria, che da soter deriva, non corrispondono sempre ai nostri «salvatore» e «salvezza» ${ }^{1}$. Questa resa ci permette tuttavia di catturare un valore semantico profondo e rivelatore che inerisce, nel corso del tempo, ai due vocaboli. Nel corso del tempo, ho precisato. Occorre tentare allora una periodizzazione: da quando soter e soteria divengono vocaboli di bruciante attualità nel panorama della cultura greca e non investono più solo il piano divino, del soccorso richiesto in circostanze difficili ai numi (salvatori) $)^{2}$, ma anche il piano umano, dell'atto di fiducia incondizionatamente riposta in un individuo eccezionale dinanzi a una crisi senza apparente via di uscita? Siamo inconsapevolmente portati a ritenere che soter, e soteria insieme con soter, costituiscano le cifre peculiari dell'età moderna della storia greca - dell'ellenismo -, un portato della sua trasformazione in senso carismatico. $\mathrm{Ma}$, a guardar meglio, questa armatura fattuale e ideologica, che si dispiegherà appieno dopo Alessandro, è ben presente nella realtà del mondo greco almeno dalla seconda metà del $\mathrm{V}$ secolo. Se non già dai decenni, o dalla rivisitazione dei decenni, in cui incombeva la minaccia persiana e i Greci hanno ottenuto quasi miracolosamente la salvezza contro un nemico così forte da schiacciarli. A segnare una vera cesura sul piano qualitativo e quantitativo sono comunque gli sviluppi che si producono con la lotta cruciale per l'egemonia sull'Ellade. È allora che gli antichi equilibri si spezzano e la drammaticità delle situazioni da affrontare si fa manifesta; solo la fuoriuscita dai quadri istituzionali e mentali consueti, se del caso mediante agenti umani straordinari - il ricorso a un soter -, sembra una soluzione 
capace di porre rimedio al malessere che attanaglia la polis - rimedio coincidente in ultima analisi con la soteria. La compenetrazione relativamente armonica di politica e religione nella Città antica inizia a dissolversi: da questo momento in poi assisteremo a faticosi tentativi di ritrovare un equilibrio fra le due sfere.

Quale che sia la data della rappresentazione dell'Edipo Re sofocleo (personalmente, continuo a pensare vada collocata non lontano dalla grande pestilenza dei primi anni della guerra peloponnesiaca), vi campeggia il tema della salvezza della Città3: nei versi iniziali della tragedia (47-48) la collettività si rimette fiduciosa, si affida senza riserve, al proprio sovrano insignito dell'appellativo soter e da cui attende appunto di essere tratta in salvo nel presente come al tempo della Sfinge ${ }^{4}$. Certo, Edipo dopo il ritorno di Creonte da Delfi si rivolgerà a Tiresia appellandosi a lui, piuttosto, in quanto unico

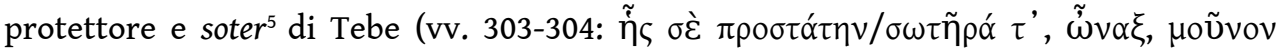
$\dot{\varepsilon} \xi \varepsilon v \rho i ́ \sigma \kappa o \mu \varepsilon v)$, quindi scoprirà come all'origine di tutto vi sia il bambino esposto, che è stato sottratto alla morte dal poimen suo salvatore (v. 1030) per esser consegnato a una sorte di sofferenze atroci quanto ineluttabili. Nel mirabolante trascolorare della figura del soter si coglie la maestria drammaturgica di Sofocle ${ }^{6}$. E l'ironia tragica rifulge proprio attraverso la formula dalla quale siamo partiti, la formula con cui il sacerdote di Tebe - Città che soffre per il male causato da Edipo - lo interpella: «la nostra terra ti chiama ora suo salvatore» ${ }^{7}$. Ciò non toglie che in questo frangente la comunità, per bocca dello hiereus, si protenda trepidante verso il proprio fastigio sperando di esserne liberata da un flagello esiziale, rimettendosi incondizionatamente a lui in qualità di soter: letta sincronicamente, la scena cui assistiamo è di un maestoso affidamento salvifico. Ho preso le mosse dall'Edipo Re, ma avrei potuto risalire piuttosto all'Antigone $e^{8}$, verosimilmente del 442.

Dall'ironia tragica all'ironia squisitamente comica. Nei Cavalieri aristofanei (si noti la programmatica composizione del Coro, che farà causa comune con il Salsicciaio alias Agoracrito), rappresentati alle Lenee del 424, soter e soteria occupano un posto senza dubbio fondamentale, ma non senza accenti beffardi: se la salvezza della polis si definisce sin dall'inizio della pièce (v. 12) come il suo vero Leitmotiv, riesce difficile non chiamare in causa i parametri dell'ironia comica quando assistiamo alla manifestazione epifanica del Salsicciaio, definito soter (vv. 146-149), o quando vengono cantate le sue lodi: «Nobilissimo corpo e anima eccelsa fra tutte, sei apparso quale salvatore per la città e noi cittadini [...]» vv. 457-458) ${ }^{9}$. L'antagonista del Paflagone (Cleone) riuscirà ad aver la meglio su di lui grazie a una sfrontatezza maggiore ed è a tale personaggio, ad Agoracrito (nome parlante: «Colui che è scelto dal popolo» o meglio «Colui che viene giudicato sull'agora»), che spetta nel finale il compito di ringiovanire miticamente (alla maniera di Medea!) Demo, entità esposta ai raggiri degli adulatori eppure occasionalmente consapevole di quanto avviene alle proprie spalle. Attraverso lo specchio deformante della commedia si conferma dunque la centralità di soter e di soteria, mentre il gioco scenico procede in una rutilante fioritura di ambivalenze: Atene necessita di essere salvata e per raggiungere l'obiettivo si ricorre a un furfante incallito, a un imbonitore incensato a causa della sua ribalderia, anzi proprio in virtù di essa capace di mettere nel sacco addirittura il tirannico Paflagone. Spesso si è postulata un'incongruenza nel plot dei Cavalieri poiché sembra difficile tener insieme la prima e la parte conclusiva della commedia, accettare la mirabolante metamorfosi di un mariuolo matricolato nel benefattore di Demo. Proviamo a concentrarci, per un istante, sulla figura del Salsicciaio. Studi recenti, che insistono sulla necessità di metter al centro del 
discorso la poetica della competizione con gli altri poeti comici, nei Cavalieri segnatamente Cratino, permettono di stabilire uno stretto parallelismo fra Agoracrito e lo stesso Aristofane ${ }^{10}$; se le cose stanno in questi termini, i Cavalieri ritrovano un'unità nell'autopromozione ${ }^{11}$ dell'autore attraverso la sua pièce - e l'acclamazione rivolta al Salsicciaio, l'attribuirgli uno statuto soterico acquistano retrospettivamente un significato pregnante: il poeta è in ultima analisi il salvatore della polis.

Con la Pace ci moviamo su un terreno molto meno insidioso molto più univoco di quello dei Cavalieri, opera (abbiamo detto) massimamente ricca di ambivalenze e quindi di insidie per chi voglia penetrarne i significati etico-politici. La commedia portata in scena alle Grandi Dionisie del 421 illustra con partecipazione simpatetica la vicenda dell'eroe comico pacifista Trigeo, che diviene il soter per eccellenza (la soteria in quanto idea-chiave è stata proposta al v. 301 e il nesso fra Pace e soteria viene sottolineato ai vv. 594-595), un soter il quale non ha beneficato solo i Greci (v. 866), ma addirittura tutti gli uomini (vv. 914-915), anche se più tardi (vv. 1035-1036) si torna a restringere la sua opera salvifica alla "città santa», Atene. Diremmo che qui del soter e della soteria si può godere senza riserve - in un abbandono che coincide con il fantasmatico volo del protagonista, compiuto paradossalmente in groppa a una creatura scatologica. Escrementizia come la scena con cui si apre la Pace. L'intelligenza comica di Aristofane è davvero senza pari: squarcia $i$ fondali e disegna collegamenti inusitati per il tramite di imprevedibili cortocircuiti. Anche nella Lisistrata, rappresentata forse alle Lenee del 411, la soteria ${ }^{12}$ è in primo piano sin dall'inizio (vv. 29-30) e l'impresa di salvare tutta la Grecia spetterà, com'è noto, alle donne: esse sole, lontane dalla logica avvizzita - di cui si fa sintomaticamente portavoce il Probulo - che vorrebbe far coincidere la soteria con le operazioni belliche, saranno capaci di salvare la Grecia (vv. 497-501, 525-526) imponendo forzosamente la pace attraverso uno sciopero sessuale. La necessità di evadere da un mondo che si infligge l'autodistruzione consegna a Lisistrata e alle sue compagne l'opportunità di un'avventura da cui verrà la fine dei mali. La soluzione, s'intende, è surreale, ma le questioni che agita Aristofane davanti al proprio pubblico sembrano tangibili. A partire dalla ricerca della soteria e dall'individuazione di un soter.

5 Seguendo questo filo rosso, dovremmo passar ad analizzare le Rane andate in scena alle Lenee del 405 e le Ecclesiazuse, di data incerta ${ }^{13}$. Ma è più importante, ai nostri fini, tornare brevemente sull'arco cronologico in cui si collocano le opere aristofanee sin qui considerate e i cui estremi coincidono appunto col 424 (data di rappresentazione dei Cavalieri) e col 411 (data di rappresentazione della Lisistrata). La domanda che occorre porsi è la seguente: che rapporto intercorre fra gli scenari salvifici disegnati da Aristofane e la realtà storica coeva? L'eroe comico (aristofaneo) ci appare una figura difficilmente riducibile a schemi prestabiliti ${ }^{14} \mathrm{e}$ ancor meno rispecchia dati di fatto per così dire certificati. Eppure, questo lungo fantastico svariare intorno alla figura del soter e all'esigenza della soteria rimanda a ciò che si muove nelle stanze della storia durante l'ultimo trentennio del $\mathrm{V}$ secolo a.C. (un analogo discorso vale, beninteso, con riguardo a Sofocle). Eccone qualche controprova.

6 Nel 424, lo stesso anno della rappresentazione dei Cavalieri, il generale spartano Brasida si dedica al vero obiettivo di una spedizione lungamente meditata: distruggere le basi dell'impero ateniese in Calcidica. Ci troviamo di fronte a un condottiero carismatico, il cui fascino promana dal valore personale, dalle straordinarie capacità strategiche e tattiche (ivi inclusa la rapidità di esecuzione dei piani militari), ma anche dal talento persuasivo - un talento persuasivo che è al servizio di un'idea sposata sino alle estreme 
conseguenze: combattere Atene dove si sente più sicura e combatterla con le sue stesse armi (la velocità, l'audacia). Attraverso le pagine di Tucidide ci è dato di toccare con mano l'impegno personale profuso da Brasida, un impegno altresì percepito dai suoi interlocutori, nel perseguire un tale progetto. Vorrei selezionare una tessera entro il mosaico della spedizione in Calcidica e quindi proporre qualche considerazione di ordine generale. Dopo la presa di Torone da parte del comandante spartano, Scione defeziona dalla lega delio-attica. Brasida vi giunge e tiene un discorso che infiamma gli Scionei; a questo punto essi lo coprono di onori pubblici e privati, come avviene per gli atleti vincitori il cui statuto è in più di un caso sovrumano (Tucidide IV 121, 1) ${ }^{15}$. La personalità di eccezione, la personalità carismatica può imprimere una svolta radicale al corso degli eventi, sino ad allora quasi prevedibile, può compiere azioni ritenute 'provvidenziali'. Vengo all'epilogo della vicenda. Sotto le mura di Anfipoli ha luogo nel 422 uno scontro determinante fra Ateniesi e Spartani; l'esito è disastroso per i primi, che perdono 600 uomini tra cui Cleone, laddove i caduti sull'altro fronte sono pochissimi ma fra costoro figura Brasida. C'è un dato che emerge dal passo di Tucidide $(\mathrm{V} 11,1)$ relativo agli onori di cui viene insignito il generale caduto in battaglia, onori che includono agoni e sacrifici annuali: la personalità del grande condottiero è sentita come elargitrice di salvezza. Anfipoli è stata salvata grazie all'impresa di Brasida che, a prezzo della propria vita, ha impedito agli Ateniesi di compiere una micidiale vendetta annunciata. La polis non basta più a sé stessa, afferma la propria dipendenza incondizionata dalle scelte di un uomo che attinge uno statuto sovrumano: essa gli deve la propria esistenza e pertanto lo riconosce come soter. La figura del soter è entrata a pieno titolo nella storia greca. Davvero molte cose sono cambiate.

7 Vengo all'altro estremo dell'arco cronologico precedentemente indicato, dunque al 411. Pochi mesi dopo la rappresentazione della Lisistrata gli oligarchi riescono a prender legalmente il potere. L'Athenaion Politeia di Aristotele o della sua scuola $(29,2)$, attingendo a fonti coeve agli eventi, ci ha trasmesso il testo del decreto di Pitodoro, con cui viene di fatto abrogata la democrazia; sarà bene riprodurlo per intero, visto l'interesse che riveste per un discorso incentrato sulla soteria. «Il popolo elegga, oltre ai dieci probuli esistenti, altri venti probuli fra coloro che abbiano superato $i$ quarant'anni: costoro, prestato giuramento di redigere le disposizioni che ritengano migliori per la polis, redigeranno disposizioni in vista della sua salvezza. Sia permesso altresì a chiunque lo voglia di avanzare proposte scritte, al fine di scegliere il meglio fra tutte». Di nuovo dall'Athenaion Politeia $(29,4)$ apprendiamo che gli eletti proposero anzitutto l'obbligo per i pritani di metter ai voti le mozioni inerenti alla salvezza di Atene. La soteria, nel cui segno si apre per così dire l'ultimo libro della Guerra del Peloponneso (dopo la catastrofe della spedizione in Sicilia, gli Ateniesi «disperavano in un simile frangente di potersi salvare» [VIII 1, 2]), è evidentemente una parola d'ordine cara agli oligarchi e il discorso attribuito a Pisandro, ancora nell'vIII libro di Tucidide (53, 2-3), costituisce una valida controprova di ciò. Che cosa significa in concreto, da parte dei 'rivoluzionari', metter al centro del proprio programma la salvezza della polis? Significa dichiarare, con una manipolazione della realtà, la bancarotta di un sistema politico - la democrazia - e proclamare la necessità di instaurarne uno alternativo. Ciò implica l'abrogazione di tutti gli istituti eretti, in progresso di tempo, a sostegno dell'ordinamento da liquidare. Che infatti scompaiono immediatamente. Quando è in questione la soteria, come si vede, ci si lascia alle spalle un intero paesaggio: se ne fuoriesce per cause di (presunta) forza maggiore. Sempre l'VIII libro di Tucidide ci permette però di constatare che quella parola d'ordine non restò appannaggio degli 
oligarchi, ma fu prontamente rivendicata anche dai democratici (vd. per esempio 75, 3). A salvare la Città possono essere proprio coloro che intendono preservarne la fisionomia consolidatasi nel corso di almeno un secolo. Si comprende l'interesse a rintuzzare la propaganda degli avversari appropriandosi del loro linguaggio politico, opportunamente capovolto. Insomma nel torno di anni in cui la soteria doveva essere molto attuale in quanto fulcro di un programma volto a ridefinire il panorama politico, la salvezza di Atene non si riduceva a un semplice slogan monopolizzato degli oligarchi. Tutt'altro: le orazioni di Lisia, recentemente riconsiderate in questa chiave, sono una vibrante testimonianza dell'utilizzazione della parola d'ordine in senso antitetico ${ }^{16}$.

È venuto il momento di porci un quesito di capitale importanza, per rispondere al quale sarebbe necessario molto più spazio di quanto io ne abbia a disposizione. La soteria, nel mondo greco del V e del IV secolo a.C., ha solo una dimensione intramondana? Proprio le Rane di Aristofane, cui ho fatto in precedenza un cursorio riferimento, ci forniscono il destro per tastare il terreno. Va ribadito che la commedia fu rappresentata dapprima ${ }^{17}$ in un periodo particolarmente difficile della storia di Atene, fra la vittoria insperata delle Arginuse (406) e la decisiva sconfitta di Egospotami (405). L'atmosfera che si respira nelle Rane, pur attraverso la potente e meravigliosa affabulazione aristofanea, è pesante. Per limitarsi a un esempio significativo, gli schiavi erano stati imbarcati sulle navi per la battaglia delle Arginuse ottenendo come ricompensa l'affrancamento, ciò che aveva molto colpito gli Ateniesi e Aristofane fra loro. La catabasi di Dioniso avviene dunque in un clima allucinato ed è a ben guardare un viaggio ultimo. Il pubblico sprofonda nella caligine dell'Ade insieme con il dio che, accompagnato dal servo Xantia, intende riportare alla luce un poeta (tragico). Il quale non sarà ovviamente, di contro $\mathrm{a}$ quanto aveva asserito Dioniso all'inizio (vv. 66-67), Euripide. Del resto il dio dichiara con parole perspicue la ragione per cui si è spinto sino agli inferi: «Sono sceso quaggiù alla ricerca di un poeta. Per quale scopo?/Perché la Città si salvi e abbia il suo teatro» (vv. 1418-1419). Si tratta dunque in senso proprio di salvare la polis e ciò che ne costituisce, noi diremmo, l'anima. La poesia, il teatro sono chiamati in causa giacché possono rigenerarla, indicando l'itinerario da percorrere. Più tardi, quando ormai si tratta di decidere chi fra Eschilo ed Euripide risalirà nell'Atene terrena con Dioniso, questi invocando Zeus Soter chiede quale via di salvezza ciascuno dei due intraveda per la Città (vv. 1433-1436). L'interrogativo, preceduto non a caso dall'altro su che cosa Eschilo ed Euripide pensino in merito ad Alcibiade (vv. 1422-1423), è capitale in tutti i sensi. Lo è certo per stabilire chi, secondo il plot, sarà al fianco del dio nella risalita. Lo è, a un livello più profondo, perché Atene sembra destinata a morire senza chi sappia consigliarla - e qui Aristofane pensa anche a sé stesso - con un'arte poetica davvero consapevole dei bisogni della polis. Aristofane insomma sta parlando della possibile fine imminente della comunità poleica con accenti sommamente preoccupati. All'interno di questa sontuosa costruzione drammaturgica si situano i versi cantati dal coro degli iniziati in cui si sono imbattuti Dioniso e Xantia durante la kathodos, versi che rivelano già la preoccupazione per le sorti di Atene. Dapprima i coreuti invitano a lodare la Soteira, quale che ne sia l'identità ${ }^{18}$, poiché ella afferma che salverà il paese in eterno (vv. 377-381); poi chiedono a Demetra, sovrana dei sacri riti, di preservare il coro che è suo (vv. 384 a-388). Ebbene, di quale soteria si parla nella fattispecie? Di una salvezza intramondana od oltremondana? L'ambientazione porta a dire che la soteria in questione è senza dubbio oltremondana, visto che i 'locutori' sono dei mystai passati a miglior vita; ciò non toglie che attraverso di loro Aristofane dia voce ai timori per il destino di Atene qui e adesso, sia pure proiettandoli in una dimensione che appunto 
non è più terrena, facendoli esprimere dagli iniziati nell'oltretomba. Così, il circolo di vita e morte si ripristina incessantemente quando è in discussione la salvezza della Città. Le formule si rivelano certo approssimative, tuttavia a me pare che le Rane ci offrano uno splendido esempio di intreccio fra salvezza oltremondana e salvezza intramondana ${ }^{19}$.

Pochi anni prima delle Rane aristofanee, nel 408, Euripide aveva messo in scena una tragedia affascinante per il modo in cui vi è descritta la condizione 'attuale' dell'eroe, ormai precipitato a un rango più che umano: l'Oreste. Il dramma trova ben presto un nucleo poetico ed emozionale nel tema della soteria ${ }^{20}$ (v. 384: «Salvami; sei giunto nel momento più propizio per le mie sciagure»; vv. 644-645: «ricchezza sarebbe se tu mi salvassi la vita, per me il più caro dei beni» [queste parole sono rivolte a Menelao sempre da Oreste]), cercata lungamente quanto vanamente nella nuova realtà in cui si muove l'eroe. Una realtà che non lascia spazio a illusioni. Privo di ogni punto di appoggio fra i membri maggiormente autorevoli del gruppo familiare e della polis, Oreste viene condannato a morte insieme con Elettra per il matricidio di cui si è macchiato, sebbene l'ordine di vendicare il padre gli fosse giunto da Apollo. L'eroe reietto può far affidamento solo sull'affetto della sorella sua compagna di sventura e sulla dedizione incondizionata dell'amico Pilade. La philia rimane l'unico valore cui aggrapparsi. Per aprirsi una strada verso la salvezza, il trio ${ }^{21}$ elabora un piano che nulla ha di eroico, ma è tout court distruttivo: uccidere Elena, rapire Ermione e usare la fanciulla come ostaggio. La soteria, sembra lecito arguirne, è irraggiungibile; i mezzi di cui l'eroe tragico, ormai rappresentante di un'umanità degradata, dispone sono grossolanamente quanto inutilmente cruenti - nel farvi ricorso si dà prova di disperazione, non ci si prepara una via d'uscita davvero praticabile - e solo l'intervento di Apollo in quanto deus ex machina pone termine all'esecuzione di un progetto che rischia di risolversi nella carneficina, restituendo a un mondo in crisi una parvenza di ordine, non sappiamo quanto duraturo e in ogni caso calato dall'alto. La salvezza, ripetiamo, resta a conti fatti una chimera, ma sarebbe comunque la meta agognata. Così si esprime Oreste nei versi che concludono la sua richiesta pressante a Menelao di un aiuto per rimanere in vita: «Ho parlato e ho chiesto la soteria, cercando ciò cui tutti anelano e non io soltanto» (vv. 678-679).

Che cos'è la salvezza a questa latitudine? Coincide con il mantenersi in vita: la vita è la posta in gioco. Siamo quasi a ridosso di un orizzonte che è quello della odierna biopolitica. La tragedia ateniese è certo uno specchio infranto, com'è stato osservato a proposito del rapporto fra tale forma letteraria e la politica coeva ${ }^{22}$, ma attraverso i frammenti dello specchio traspaiono le dinamiche laceranti che attraversano la Città. Dove, mentre infuria la più radicale delle staseis, non sembra esservi alcuno scampo. Al centro dell'Oreste euripideo v'è, infatti, l'impossibilità della soteria.

11 La diagnosi risulta chiara, se si parte dallo stato in cui versa la polis alla fine del V secolo. La soluzione ai problemi apparentemente insolubili sul tappeto può venire solo dalla presa d'atto di uno smottamento nell'assetto tradizionale delle istituzioni e dall'accettazione del nuovo corso che la realtà delle cose (e dei rapporti di forza) imprime alla storia greca. Basti pensare ad Alcibiade o ancora a Lisandro, divinizzato in vita. Insomma, il leader carismatico tante volte indicato come colui che riuscirà a traghettare dal vecchio verso un nuovo mondo è salito, sta salendo alla ribalta. Si cercano rimedi eccezionali per il corpo politico malato e ciò contribuisce senza dubbio a spiegare - sia detto per inciso - il rigoglioso fiorire del culto di Asclepio, savior non 
meno che healer: il Pluto aristofaneo, di cui possediamo la redazione del 388 (la precedente rimonta al 408), è una pietra miliare in questo itinerario.

Non stupisce che, in un simile quadro, prenda piede la riflessione politico-filosofica sulla regalità e sul re ideale ${ }^{23}$. Il suo sviluppo si coglie lungo i decenni iniziali del IV secolo a.C. Quando (intorno al 374/373) ${ }^{24}$ Isocrate scrive l'A Nicocle, che tanta importanza avrebbe avuto in epoca successiva come speculum principis, si premura di rivolgere al successore di Evagora un suggerimento che concerne proprio la soteria dei sudditi: «Non voler affermare la tua autorità con la durezza e col rigore dei castighi, ma facendo sentire a tutti la superiorità della tua intelligenza e instillando in loro la convinzione che sei in grado di provvedere alla loro salvezza, alla loro salvaguardia

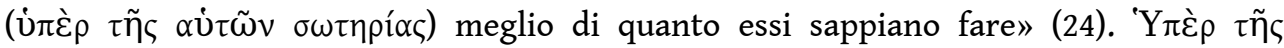
$\sigma \omega \tau \eta{ }^{\prime} \alpha \varsigma$ appare un'espressione di uso corrente: vi ritornerò fra breve. Ciò che risulta notevole, nella raccomandazione di Isocrate a Nicocle, è la circostanza che, all'interno di rapporti di forza evidentemente asimmetrici, il sovrano si mostri in grado di provvedere alla soteria di chi è a lui sottoposto ed esibisca questa sua qualità. La presenza di una formula di beneficio, avente a oggetto la salvezza di quanti dipendono dal re, sembra davvero un annuncio dei tempi futuri; la letteratura in quanto espressione dell'immaginario anticipa, come sempre, sviluppi (schemi di pensiero e di comportamento o di propaganda) poi destinati a divenire moneta corrente nella realtà della storia ellenistica.

Dicevo poc'anzi che ن் $\pi \grave{\varepsilon} \rho \tau \tilde{\eta} \varsigma$ $\sigma \omega \tau \eta \rho i ́ \alpha \varsigma$ è un'espressione di uso corrente; ancora più frequente è quella senza l'articolo determinativo, u̇đè $\sigma \omega \tau \eta i^{\alpha} \alpha \varsigma$. Quando l'esistenza individuale e collettiva si va facendo precaria, la sfera salvifico-salutare assume un rilievo sempre più marcato. Partendo da ن் $\dot{\varepsilon} \rho \tau \eta \tilde{\zeta} \sigma \omega \tau \eta \rho i ́ \alpha \varsigma$ e dall'ancor più frequente

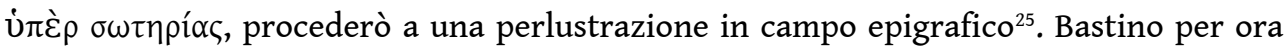
due soli esempi. Una legge forse risalente al 354/353-343, ma reiscritta verso il 200 a.C. ${ }^{26}$, ha giustamente attirato l'attenzione degli studiosi dopo la sua prima - parziale pubblicazione (SEG LII, 2002 [2006], 104); l'esordio dell'iscrizione è sintomatico, in quanto mira a garantire che "tutto sia sicuro e integro nel santuario della dea di

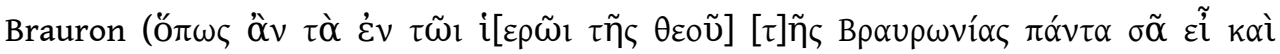
ن̀y(n))», a partire dal tempio: l'elenco dettagliato delle costruzioni interessate alle misure da prendere si conclude con la locuzione riassuntiva, o comunque onnicomprensiva, «e ogni edificio che la polis ha eretto e dedicato alla dea per la salvezza (ن் $\dot{\varepsilon} \rho \sigma \omega \tau \eta \rho i ́ \alpha \varsigma)$ del popolo di Atene». Se la legge rimonta effettivamente alla metà del IV secolo, si possono ben comprendere le ragioni per cui gli Ateniesi utilizzarono parole dalle quali traspare una viva preoccupazione e ricorsero alla divinità in cerca della soteria - la guerra con gli alleati (la «guerra sociale» del 357-355) si era appena conclusa, si profilava lo scontro con la Macedonia ed era ovvio rivolgersi, in cerca di rassicurazione, al santuario della dea dove seguendo il normale iter iniziatico le fanciulle ateniesi si forgiavano per apprestarsi a dare nuova linfa alla comunità. La reiscrizione della legge in anni che sembrerebbero essere quelli, tormentati, della seconda guerra macedonica (con la sua scia di ingenti distruzioni) sarebbe stata a sua volta più che giustificata. $\mathrm{Al} \mathrm{306/305}$ va datato invece il decreto in onore di Timostene di Caristo, di cui fra l'altro si ricordano le benemerenze acquisite presso gli Ateniesi durante il conflitto contro Antipatro, quando inviato come delegato della sua polis

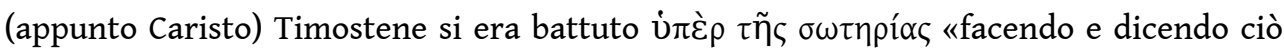
che era vantaggioso per il koinon dei Greci e per i Caristii» (IG II 467 [con Addendum a p. 
661]). Di nuovo la situazione segnalata dalla formula di beneficio «per la salvezza»si rivela particolarmente critica: il riferimento è alla guerra lamiaca (323-322) ${ }^{27}$.

Ampliando il nostro giro di orizzonte a una formula vicina a $\dot{u} \pi \dot{\varepsilon} \rho \tau \tilde{\eta} \varsigma \sigma \omega \tau \eta \rho i ́ \alpha \varsigma$,

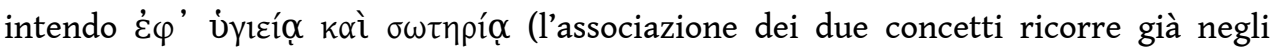
Uccelli aristofanei [878-879]), l'impressione che ricaviamo non è diversa. Sinora la sua prima attestazione risale al 343/342: in un testo epigrafico particolarmente intricato (IG II $\left.^{3} 1,306\right)$ si susseguono addirittura cinque psephismata, di cui uno contiene la proposta - ne è autore Cefisofonte - di insignire la boule di una corona aurea per aver sovrainteso all'ordinato svolgimento delle feste per Dioniso (decreto 1), mentre un altro decreto ci permette di ricostruire i divisamenti dell'attidografo Fanodemo, il quale invita a dedicare una statua a Efesto e ad Atena Hephaistia, a riportarvi i nomi, i patronimici e i demotici dei buleuti; segue il riferimento a un atto verosimilmente sacrificale compiuto dagli stessi buleuti «per la salute e la salvezza della boule e del demos degli Ateniesi»; infine Fanodemo consiglia di iscrivere sulla statua il decreto in base al quale la boule aveva ricevuto la corona (decreto 2). Non mi inoltro nella disamina di un documento complesso per la sua stratificazione interna (il decreto 3 riferisce la proposta di Dinostrato: poiché la boule ha deciso di onorare chi ha parlato e agito nel modo migliore, poiché la boule ha votato e scelto Fanodemo, che la stessa boule disponga di premiarlo con una corona del valore di 500 dracme, mentre l'assemblea potrà a sua volta attribuirgli, in riconoscimento dei suoi meriti, una corona del valore di 1000 dracme; nei decreti 4 e 5 si deliberano onori per Eudosso, amministratore della boule), ma vorrei ricordare quanto sia cruciale il torno d'anni in cui si inquadra il

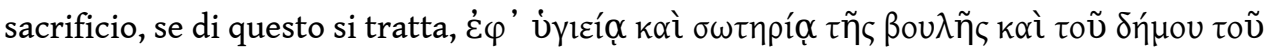
A $\theta \eta v \alpha i ́ \omega v$. Siamo alla vigilia dell'organizzazione della lega ellenica contro Filippo II. Di lì a poco, per l'esattezza nel 332/331, sempre Fanodemo impiegherà per ben due volte la formula "per la salute e la salvezza» quando si tratterà di sancire a tutti gli effetti l'appropriazione da parte ateniese del santuario di Anfiarao a Oròpo, incoronando il dio $\left(I G I^{3} 1,349\right)^{28}$. Una formula salutare e salvifica per una divinità salutare e salvifica, si sarebbe portati a dire. Ma è ormai tempo di concludere questa digressione ${ }^{29}$ che ci ha permesso di toccare con mano, attraverso l'esame di un modesto campione di iscrizioni, quanto l'esigenza della soteria si stia facendo sempre più attuale in anni convulsi. Torniamo dunque al nostro filo conduttore principale e ricolleghiamoci a ciò che dicevamo traendo spunto dall'A Nicocle di Isocrate.

15 Allorché Senofonte, in cui è preminente la riflessione sulla regalità come forma ideale di governo capace di condurre un mondo in crisi verso lidi più sicuri, redige l'Agesilao (dopo la morte del re, nel 360/359), non può esimersi dall'introdurre un encomio nell'encomio, ricordando fra l'altro come «coloro che correvano con lui qualche pericolo [lo chiamavano] salvatore dopo gli dèi» $(11,13)^{30}$. I termini in cui si esprime Senofonte meritano di esser attentamente scrutinati. Ne emerge il vincolo di incondizionata devozione dei commilitoni - di costoro sembra trattarsi - nei confronti del sovrano, il fascino che evidentemente egli esercitava su di loro; ne emerge altresì il rispetto degli standard della religiosità tradizionale, che impone di collocare un uomo anche se molto valente in secondo piano, rispetto agli dèi. Circa questo secondo aspetto, mi pare che nelle parole senofontee si percepisca una polemica sotto traccia contro la divinizzazione in vita di un uomo, onore che era stato accettato di buon grado da Lisandro, ma che Agesilao sembrerebbe aver nettamente rifiutato ${ }^{31}$. Per quanto il sovrano e i suoi contubernali dimostrino un ossequio forse non formale verso 
la pietas avita in base ai cui dettami gli dèi vanno venerati in sommo grado e nessuno dei viventi può aspirare a competere con loro, rimane comunque il fatto che l'appellativo soter è documentato a chiare lettere per Agesilao da Senofonte. Nel re spartano si esprime un potere di segno carismatico, sentito come capace di tenere avvinti a chi lo detiene quanti gravitano intorno a lui. Prima di lasciarci alle spalle l'encomio di Agesilao, vorrei segnalare una lacuna di non trascurabile consistenza nelle nostre conoscenze. È ammissibile, ma non certo, che Ione di Chio (F 90 Leurini) all'inizio di un'elegia simpotica si rivolgesse con l'appellativo «salvatore e padre ( $\sigma \omega \tau \eta ́ \rho$ $\tau \varepsilon \pi \alpha \tau \eta ́ p ~ \tau \varepsilon) »$ a un sovrano di Sparta: si è suggerito, nella fattispecie, il nome di Archidamo (II). In effetti, l'individuazione della figura che il poeta subito dopo designa

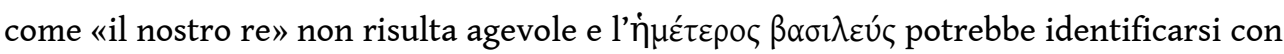
il dio che presiede al simposio, insomma con Dioniso ${ }^{32}$. Ove si voglia sottoscrivere l'ipotesi secondo cui il destinatario dell'allocuzione di Ione sia piuttosto un personaggio umano, resta da stabilire sino a che punto nella Sparta di età arcaica e classica il potere tradizionale (qui segnalato da $\pi \alpha \tau \eta ́ p$ ) e quello carismatico (cui fa riferimento $\sigma \omega \tau \eta ́ p$ ), per riprendere la celebre nomenclatura weberiana, si confondessero; resta da stabilire soprattutto quale sfondo epicorio presupponga l'attribuzione ad Agesilao della qualifica di soter, documentata da Senofonte.

Sarebbe superfluo vagliare una volta di più le due fonti, rispettivamente Diodoro e Plutarco, che forniscono preziose informazioni sul rientro prima nel 357 e poi nel 356/355 di Dione nella Siracusa di Dionisio II. Senza dubbio esistono alcune divergenze fra il racconto di Diodoro (che deriverebbe probabilmente, o comunque prevalentemente, da Eforo) ${ }^{33}$ e quello plutarcheo (dove si nota, a quanto pare, soprattutto la presenza di Timonide di Leucade, senza perciò escludere un apporto timaico) circa gli onori di cui viene fatto oggetto Dione: se il biografo tende ben presto ad accreditare il carattere divino o quasi-divino di quegli onori (Dione 29, 1-2; 46, 1), tributati in un clima di esaltazione collettiva, lo storico parrebbe attento a ricostruire in modo più sobrio e circostanziato il corso degli eventi (XVI 11, 1-2; 20, 5-6), sottolineando come la stessa assemblea che nel 356/355 designò Dione stratego con

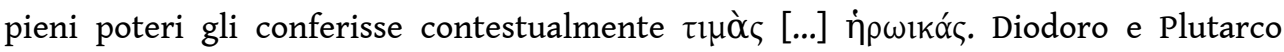
risultano tuttavia in accordo, è stato debitamente rilevato ${ }^{34}$, per quanto concerne l'attribuzione dello status di soter a Dione. Leggiamo infatti in Diodoro: «I Siracusani [...]

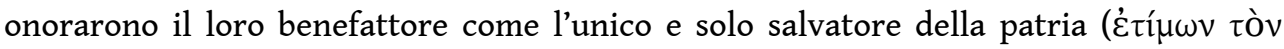

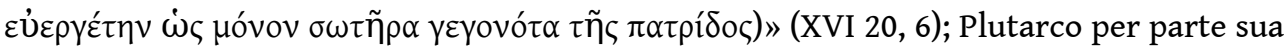

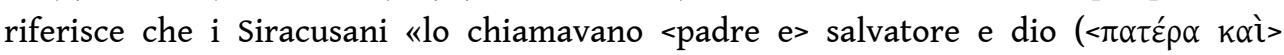

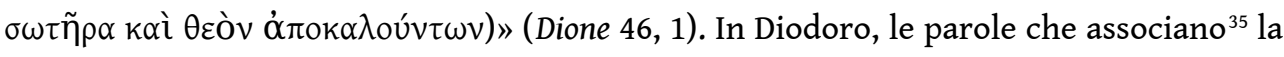
qualifica di euergetes e di soter - combinazione molto frequente in età ellenistica suggellano per così dire l'intera sezione dionea del XVI libro della Biblioteca storica, laddove in Plutarco i termini "padre e salvatore e dio» ci restituiscono o sembrano restituirci un'acclamazione con cui viene accolto Dione all'atto del suo reingresso a Siracusa: in ogni caso, non v'è ragione di dubitare che egli fosse riconosciuto come soter dalla comunità. Per completare il quadro, occorre aggiungere che Diodoro dà conto di una serie di atti sacrali compiuti da Dione prima della convocazione dell'assemblea, fra

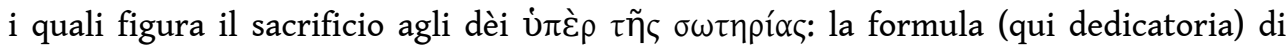
beneficio impiegata è comunque indicativa e ha già attirato la nostra attenzione in precedenza. Il titolo di soter, l'idea-guida della soteria ineriscono alla figura storica di Dione. 
17 C'è un'interferenza che dovrebbe esser presa in considerazione a questo proposito, perché il liberatore di Siracusa dalla tirannide è legato a doppio filo a Platone e all'Accademia, perché in terra di Sicilia qualcuno cerca nonostante tutto di tradurre in atti politici concreti disegni a lungo meditati in sede di riflessione teorica ${ }^{36}$. Nel grande plesso formato dai libri V-VI della Repubblica si profila nitidamente il tema della soteria - e dei soteres - della polis da fondare o di cui va garantita la politeia e quel tema si intreccia, in qualche misura, con il ruolo affidato a dynastai come Dione. Considerevole specialmente il passo in cui Socrate rileva come basti che uno fra i figli di re o di dynastai dotati di natura filosofica si sia salvato e che stabilisca le leggi e le pratiche di vita idonee alla costruzione della kallipolis perché i cittadini accettino di seguirlo, facendo giungere a compimento quanto sembra incredibile (502 a 5-b 8) ${ }^{37}$. Si noti che di lì a poco ci si riferisce ai filosofi come a «i salvatori dell'ordinamento politico» fondato (502 c 9-d 3): perché vi sia salvezza della kallipolis devono esservi dei soteres a ciò espressamente preposti. Procediamo oltre. Al centro del Politico, redatto verso il 360 o poco dopo, figura il rapporto fra uomo politico dotato di arte regia e 'governo' delle

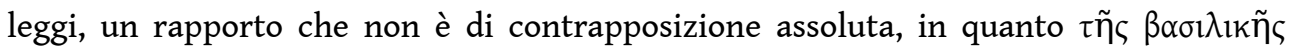

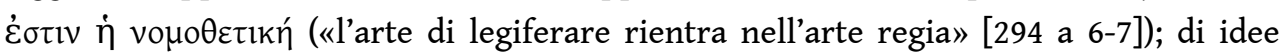
siffatte dovevano esser imbevuti uomini quali Dione. Ma bisogna guardare anche al di là del Politico. Quello della salvezza è un filo rosso che attraversa da un capo all'altro i Nomoi platonici ${ }^{38}$ e nelle pagine conclusive dell'opera pubblicata postuma il discorso

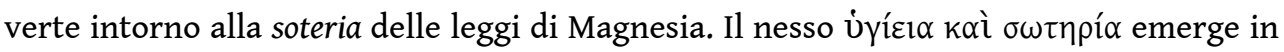
un punto saliente del testo (a partire da 960 b), là dove si tratta di assicurare l'immutabilità dei nomoi stabiliti per Magnesia. Il passo merita di esser citato per esteso: «queste cose [il periodo che precede fa riferimento alle Parche ed è uno dei più problematici, dal punto di vista critico-testuale, delle Leggi ${ }^{39}$ ] devono procurare alla

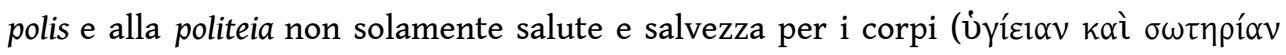

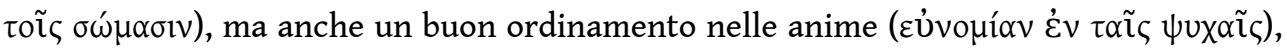
soprattutto (queste cose devono procurare) la soteria delle leggi ( $\sigma \omega \tau \eta \rho i ́ \alpha v ~ \tau \tilde{\omega} v$ vó $\mu \omega v)$ ». Lo scenario appare suggestivo. È notevole, a mio avviso, da un lato la ricorrenza dell'espressione "salute e salvezza per i corpi» - ciò che permette di

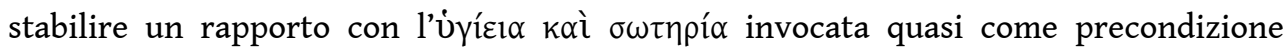
indispensabile della vita associata in alcune iscrizioni in cui figura quella formula ${ }^{40}-$, dall'altro la scelta di un vocabolo come soteria per indicare l'inalterabilità delle leggi di Magnesia. Vorrei qui riproporre quanto ha scritto in una bella monografia Christine Hayes: «Che cosa assicura la qualità stabile e irreversibile della legge e così funge da suo salvatore? Delle persone che posseggono un'educazione conveniente, una fede nell'anteriorità sempiterna dell'anima e nella sua sovranità sul mondo dei corpi, un desiderio di discernere la ragione o logos che ordina i cieli (Leggi 964d-969c). Noti come il "Consiglio Notturno", questi guardiani vigilano sullo Stato e, assicurando la salvezza della legge, assicurano la salvezza dello Stato» ${ }^{41}$. La parabola del pensiero platonico dalla Repubblica alle Leggi è, penso, in grado di illuminare almeno in parte l'idea-guida della soteria così strettamente connessa alla figura di Dione. Scendendo su un terreno ancora più concreto e con ogni probabilità vicino agli eventi occorsi a Siracusa, l'autore della Settima Lettera (336 b 7-337 d 2) suggerisce di por termine alla guerra civile, di fissare leggi eguali per tutti, leggi che proprio i vincitori del momento dovranno stabilire e far osservare; rivolgendosi ancora alla fazione che ha avuto la meglio egli la sprona a scegliere, se vuole davvero la salvezza della polis, quanti fra i Greci hanno fama di esser i migliori, ad attirarli a Siracusa con preghiere e grandissimi onori: costoro, 
prestato giuramento, vareranno leggi che non favoriscano nessuno - una volta che esse siano state fissate, tutto dipenderà dalla loro scrupolosa osservanza specie da parte dei vincitori; ove questa condizione venga rispettata, ne deriveranno soteria, felicità e liberazione da tutti i mali. La salvezza della polis, in un tutt'uno con l'ossequio per le leggi fissate da chi è davvero competente in materia, si inscrive perfettamente nella riflessione politologica di Platone e forse non siamo troppo lontani dal vero ipotizzando che Dione si sentisse o fosse stato chiamato a contribuire, per la sua parte, alla realizzazione di una sorta di programma ideale ${ }^{42}$. Dipende anche da ciò la sottolineatura dei caratteri soterici nella figura e nell'attività di questo esponente dell'Accademia? D'altronde i sodali di Dione non avranno avuto difficoltà, crediamo, a far risuonare la parola d'ordine della soteria (o altre consimili) quando il loro leader ritornò a Siracusa per liberarla dalla tirannide e da quell'arena potrebbe esser rifluita, attraverso fonti vicine al corso degli eventi, sino a Diodoro e a Plutarco. Non intendo minimamente affermare che un concetto come soteria sia stato appannaggio esclusivo (o privilegiato) dell'Accademia, in sede di elaborazione politologica; propongo solo alla comune attenzione la possibilità che dalla scuola di Platone sia venuta un'ulteriore sollecitazione a connotare l'operato di Dione attraverso Schlagwörter di uso comune in situazioni di crisi e tuttavia rilanciati da parte dell'Accademia all'atto di indicare una forma di monarchia idealizzata quale via d'uscita dai problemi che affliggevano la polis.

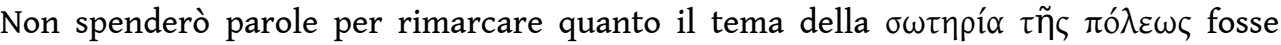
disponibile ancora nell'età di Demostene, che nell'orazione Per la corona (169 ss.; vd. poi 248) sa accreditare il proprio ingresso in scena, data la drammaticità della situazione cui si trova davanti Atene dopo la conquista di Elatea per mano di Filippo II (339 a.C.),

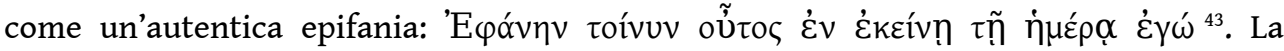
manifestazione del soter in vista della salvezza della polis è dunque sempre attuale: sebbene il Salsicciaio dei Cavalieri aristofanei fosse un salvatore sui generis, anche il suo intervento soterico assumeva i caratteri di un'epifania.

Quando si svolge il magistero di Aristotele ${ }^{44}$, la crisi dell'istituto poleico ha ormai, dunque, una lunga storia: il disagio si è talmente stratificato che la stessa parola d'ordine che lo denunciava, soteria, ha perso in qualche misura la propria carica emergenziale ed è suscettibile di esser assunta come un indicatore linguistico quasineutro; soteria entra a far parte di una nomenclatura standardizzata (in coppia antitetica con phthora) e Aristotele vi fa ricorso analizzando la realtà degli ordinamenti politico-istituzionali, inventariandoli. Ma la descrizione non si esaurisce in sé stessa. All'analisi 'scientifica' della realtà degli ordinamenti politico-istituzionali, si sovrappongono i suggerimenti del filosofo per correggere le storture che gli accade di diagnosticare specialmente confrontandosi con regimi lontani dall'ottimo, suggerimenti elargiti in nome del realismo per assicurare la loro stabilità. Che è un bene in sé.

Un'analisi dedicata al concetto di soteria nell' Etica Nicomachea e nella Politica non dovrebbe tuttavia limitarsi a rilevare solo la presenza dell'istanza terapeutica accanto alla ricognizione diagnostica, fondata sull'empiria. Soteria è infatti anche un'idea-limite e come tale detta delle coordinate ideali o ideologiche. Che si tratti del rapporto necessitato padrone-schiavo $\mathrm{o}$ della polis in quanto pluralità imperniata sull'uguaglianza come reciprocità o dell'indispensabile condivisione di còmpiti fra agenti umani diversi entro una comunità coincidente con la politeia, il concetto di soteria determina le condizioni basilari in assenza delle quali non v'è preservazione nel 
segno dell'integrità. Soteria sta anche a demarcare negli scritti aristotelici i confini di un mondo sentito come pienamente dotato di senso e fra i segnacoli di quel mondo c'è in primo luogo la continuità dell'istituto della polis, da salvaguardare a ogni costo.

21 La disamina che ho cercato di compiere dimostra, a me pare, quanto profonda, pervasiva, sia la presenza di soter e soteria nei variegati scenari del V e del IV secolo. Sotto l'impulso di una lotta senza sosta per l'egemonia sull'Ellade entra in crisi il modello tradizionale della Città, in cui la comunità nel suo insieme appare arbitra del proprio destino e la politica si fonde indissolubilmente con la religione: la svolta carismatica è in stretto rapporto con quello scompaginamento. L'evocazione di un salvatore, il primato riconosciuto alla salvezza costituiscono una manifestazione palpabile del nuovo corso della storia greca e non sorprende che in più di un caso il salvatore e la salvezza assumano una coloritura anche religiosa, nel tentativo di ricomporre sia pure faticosamente ciò che si va disaggregando, l'istanza politica e l'istanza religiosa dapprima fuse, abbiamo visto, in un insieme 'organico'. Almeno sotto un certo profilo (e semplificando in massimo grado), il punto di arrivo del processo in atto è rappresentato dalle dediche votive in cui il nome del sovrano regnante viene seguito dall'epiteto Soter o meglio ancora è abbinato al nome della divinità, con cui può condividere sintomaticamente questa epiclesi cultuale. Il re è a tutti gli effetti un dio celebrato in quanto salvatore. Ma così siamo giunti davvero all'età ellenistica.

\section{BIBLIOGRAFIA}

BEARzoT 2013: C. Bearzot, «Soteria oligarchica e soteria democratica tra 411 e 404», in N. Cusumano, D. Motta (a cura di), Xenia. Studi in onore di L. Marino, Caltanissetta-Roma 2013, 113-122.

BILES 2011: Z.P. Biles, Aristophanes and the Poetics of Competition, Cambridge 2011.

BURIAN 1974: P. Burian, «Suppliant and Saviour: Oedipus at Colonus», Phoenix 28 (1974), 408-429.

CAMASSA 2016: G. Camassa, «Statuto del corpo e annuncio di salvezza: prolegomeni», in Studi Ellenistici, XXX, Pisa-Roma 2016, 259-289.

CAMASSA 2018: G. Camassa, «Riflessioni sul concetto di soteria in Aristotele, a partire dalla Politica», in C. Bearzot, M. Canevaro, T. Gargiulo, E. Poddighe (a cura di), Athenaion Politeiai tra storia, politica e sociologia: Aristotele e Pseudo-Senofonte, Milano 2018, 87-103.

CANEVARo 2011: M. Canevaro, «The Twilight of Nomothesia: Legislation in Early-Hellenistic Athens (322-301)», Dike 14 (2011), 55-85.

CARTLEDGE 1987: P. Cartledge, Agesilaos and the Crisis of Sparta, Baltimore 1987.

CHRISTODoulou 2016: P. Christodoulou, «Philosophie et pouvoir royal dans la pensée d'Isocrate et de Platon. Le règne de l'expert politique», Cahiers du Centre Gustave Glotz 27 (2016), 137-169.

EUCKEN 1983: C. Eucken, Isokrates. Seine Positionen in der Auseinenadersetzung mit den zeitgenössischen Philosophen, Berlin-New York 1983. 
FARAONE 1997: C.A. Faraone, «Salvation and Female Heroics in the Parodos of Aristophanes' Lysistrata», Journal of Hellenic Studies 117 (1997), 38-59.

FLOWER 1988: M.A. Flower, «Agesilaus of Sparta and the Origins of the Ruler Cult», Classical Quarterly N.S. 38 (1988), 123-134.

Goukowsky 2016: P. Goukowsky, «Introduction», in Diodore de Sicile, Bibliothèque historique. Tome XI, Livre XVI. Introduction et notes de P. Goukowsky, texte établi par D. Gaillard-Goukowsky, traduit par P. Goukowsky, Paris 2016, VII-CCXL.

GRAF 2017: F. Graf, «Theoi Soteres», Archiv für Religionswissenschaft 18-19 (2017), 239-253.

HAAKE 2020: M. Haake, «The Academy in Athenian Politics and Society», in P. Kalligas, C. Balla, E. Baziotopoulou-Valavani, V. Karasmanis (Eds.), Plato's Academy. Its Workings and Its History, Cambridge 2020, 65-88.

HAYES 2015: C. Hayes, What's Divine about Divine Law? Early Perspectives, Princeton-Oxford 2015.

HORNBLOWER 2004: S. Hornblower, A Commentary on Thucydides, II. Books IV-V.24, rist. Oxford 2004.

JiM 2015: T.S.F. Jim, «Can Soteira be Named? The Problem of the Bare Trans-Divine Epithet», Zeitschrift für Papyrologie und Epigraphik 195 (2015), 63-74.

JiM 2017: T.S.F. Jim, «'Salvation’ (Soteria) and Ancient Mystery Cults», Archiv für Religionswissenschaft 18-19 (2017), 255-281.

KATSAROS 2007: A. Katsaros, «Staging Empire and Other in Ion's Sympotica», in V. Jennings, A. Katsaros (Eds.), The World of Ion of Chios, Leiden-Boston 2007, 217-240.

KLEINKNECHT 1937: H. Kleinknecht, «Zur Parodie des Gottmenschentums bei Aristophanes», Archiv für Religionswissenschaft 34 (1937), 294-313.

KLosкo 2006: G. Klosko, The Development of Plato's Political Theory², Oxford 2006.

LAMBERT 2018: S.D. Lambert, Inscribed Athenian Laws and Decrees in the Age of Demosthenes. Historical Essays, Leiden-Boston 2018.

MAGNelLi 2017: E. Magnelli, «Rethinking Aristophanes' Comic Hero: Utopianism, Ambiguity, and Athenian Politics», Polis. The Journal for Ancient Greek Political Thought 34 (2017), 390-404.

MENN 2013: S. Menn, «Plato's Soteriology?», in V. Adluri (Ed.), Philosophy and Salvation in Greek Philosophy, Berlin-Boston 2013, 191-216.

Mitchell 2013: L. Mitchell, The Heroic Rulers of Archaic and Classical Greece, London-New Delhi-New York-Sydney 2013.

MORGAN 2015: K.A. Morgan, Pindar and the Construction of Syracusan Monarchy in the Fifth Century B.C., Oxford 2015.

Muccioli 1997: F. Muccioli, «I Siracusani, Dione e l'Herrscherkult», in L. Criscuolo, G. Geraci, C. Salvaterra (a cura di), Simblos. Scritti di storia antica, II, Bologna 1997, 107-133.

Muccioli 1999: F. Muccioli, Dionisio II. Storia e tradizione letteraria, Bologna 1999.

NAGY 2013: G. Nagy, The Ancient Greek Hero in 24 Hours, Cambridge (Mass.)-London 2013.

NeLSON 2016: S. Nelson, Aristophanes and His Tragic Muse. Comedy, Tragedy and the Polis in 5th Century Athens, Leiden-Boston 2016.

OLIVER 2007: G.J. Oliver, War, Food, and Politics in Early Hellenistic Athens, Oxford 2007. 
OSBORNE 1981: M.J. Osborne, Naturalization in Athens, I. A Corpus of Athenian Decrees Granting Citizenship, Brussel 1981.

OSBORNE 1982: M.J. Osborne, Naturalization in Athens, II. Commentaries on the Decrees Granting Citizenship, Brussel 1982.

PAQUET 1973: L. Paquet, Platon. La médiation du regard. Essai d'interprétation, Leiden 1973.

PARKER 2017: R. Parker, Greek Gods Abroad. Names, Nature, and Transformations, Oakland 2017.

PrausCello 2014: L. Prauscello, Performing Citizenship in Plato's Laws, Cambridge 2014.

REBENICH 2017: S. Rebenich unter Mitarbeit von J. Wienand (Hrsg.), Monarchische Herrschaft im Altertum, Berlin-Boston 2017.

REINEKE 2014: M.J. Reineke, Intimate Domain. Desire, Trauma, and Mimetic Theory, East Lansing 2014.

RosenbloOM 2012: D. Rosenbloom, «Democracy and Its Discontents in Late Fifth-Century Drama», in A. Markantonatos, B. Zimmermann (Eds.), Crisis on the Stage. Tragedy and Comedy in Late FifthCentury Athens, Berlin-Boston 2012, 405-442.

SANDVoss 1971: E. Sandvoss, Soteria. Philosophische Grundlagen der platonischen Gesetzgebung, Göttingen-Zürich-Frankfurt 1971.

SCAFURO 2009: A.C. Scafuro, «The Crowning of Amphiaraos», in L. Mitchell, L. Rubinstein (Eds.), Greek History and Epigraphy. Essays in Honour of P.J. Rhodes, Swansea 2009, 59-86.

SCHÖPSDAU 2011: K. Schöpsdau, Platon, Nomoi (Gesetze). Buch VIII-XII. Übersetzung und Kommentar von K.S., Göttingen-Oakville 2011.

SCHоFIELD 1999: M. Schofield, Saving the City. Philosopher-Kings and Other Classical Paradigms, LondonNew-York 1999.

SLATER 1988: W.J. Slater, «The Epiphany of Demosthenes», Phoenix 42 (1988), 126-130.

TORDOFF 2017: R. Tordoff, «Memory and the Rhetoric of $\sigma \omega \tau \eta p i ́ \alpha$ in Aristophanes' Assembly Women », Histos Supplement 6 (2017), 143-210.

TRAMPEDACH 1994: K. Trampedach, Platon, die Akademie und die zeitgenössische Politik, Stuttgart 1994. VALERIO 2013: F. Valerio, Ione di Chio. Frammenti elegiaci e melici, Bologna 2013.

VEGETTI 2005: M. Vegetti, Il tempo, la storia, l'utopia, in Platone, La Repubblica. Traduzione e commento a cura di M.V., VI. Libro VIII-IX, Napoli 2005, 137-168.

VIDAL-NAQUET 2002: P. Vidal-Naquet, Lo specchio infranto. Tragedia ateniese e politica (ed. orig. Paris 2001), trad. it., Roma 2002.

WoHL 2015: V. Wohl, Euripides and the Politics of Form, Princeton-Oxford 2015.

ZIMMERMANN 2014: B. Zimmermann, «Aristophanes», in M. Fontaine, A.C. Scafuro (Eds.), The Oxford Handbook of Greek and Roman Comedy, Oxford 2014, 132-159.

\section{NOTE}

1. Avverto sin da ora che ho limitato al minimo i rinvii bibliografici. Qualche punto di riferimento su soter e soteria per esempio in CAMASSA 2016; sull'argomento è in preparazione uno studio d'assieme di T.S.F. Jim (cfr. PARKER 2017, 146 con nota 81): alcuni contributi della studiosa che preludono alla sua monografia sono citati infra. 
2. Vd. ora in proposito GRAF 2017.

3. Che è, vorrei precisarlo, l'argomento di cui mi occupo nell'arco di queste pagine. Si tratta dunque della salvezza e del salvatore della polis e non, poniamo, dell'ethnos.

4. Secondo NAGY 2013, 534-535 l'epiteto soter per Edipo sarebbe «fuori posto» in questo contesto.

5. Questo 'spostamento' del ruolo di soter è preparato, alla fine del prologo, dalle parole del sacerdote: «È Febo a mandarci queste profezie - che sia lo stesso Febo il salvatore e il medico del male» (vv. 149-150).

6. Al culmine della vicenda (vv. 1179-1180, 1455-1458) appare, non a caso, il verbo $\sigma \omega ́ \zeta \zeta$.

7. L'Edipo a Colono delinea un'immagine speculare a quella dell'Edipo Re - l'eroe è un supplice che diviene soter per chi lo accoglie: vd. specialmente BURIAN 1974. Tuttavia, il quadro è forse più complesso di quello qui sinteticamente delineato e il soter non cancella il contaminatore: un tentativo di bilancio in REINEKE 2014, 126-129 con le note pertinenti (ove bibliografia fondamentale).

8. Molto significativi ai nostri fini i versi 184-191 (l'antonimo di soteria è qui ate), 1058.

9. KLEINKNECHT 1937, partic. 306-308.

10. Riferimenti bibliografici per esempio in NELSON 2016, 195 nota 51; inoltre BILES 2011, 97-133.

11. Non senza accenti istrioneschi...

12. Sulla centralità della soteria nella Lisistrata, vd. FARAONE 1997.

13. Mi soffermerò in seguito sulle Rane, ma non sulle Ecclesiazuse che sono state attentamente scrutinate da TORDOFF 2017.

14. ZIMMERMANN 2014, 145-147; inoltre, MAGNELLI 2017.

15. Vd. anzitutto HORNBLOWER 2004, 380-385 (nonché viii), con la bibliografia anteriore.

16. Per quanto precede, si terrà presente BEARZOT 2013 (con bibliografia).

17. Una nuova redazione delle Rane rimonta al 405/404.

18. Vd. ora al riguardo Jiм 2015, 64 (con status quaestionis nelle note pertinenti), 74 .

19. Secondo Jiм 2017 , soteria indica dalle prime attestazioni del termine sino al IV secolo d.C. una salvezza fondamentalmente intramondana.

20. Sulla soteria nelle Rane e nell'Oreste, vd. in anni a noi vicini RoSENBLOOM 2012; inoltre (con riguardo alla tragedia euripidea) WoHL 2015, 118-131.

21. Non mi sembra troppo azzardato ammettere che ci troviamo qui di fronte a una sorta di trasposizione dei rapporti esistenti entro un'eteria.

22. VIDAL-NAQUET 2002.

23. Un punto di riferimento è adesso REBENICH 2017. Vd. anche, sul periodo che qui interessa, CHRISTODOULOU 2016.

24. EUCKEN 1983, 215.

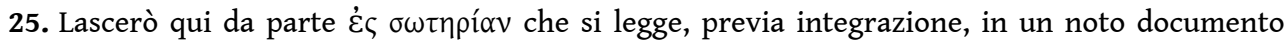
epigrafico del 405/404 a.C.: IG I ${ }^{3}$ 125. Più comune, nelle iscrizioni dei secoli successivi, la formula

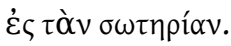

26. LAMBERT 2018, 42 nota 78 (continuazione dalla pagina precedente), 166 nota 29. Vd. pure CANEVARo 2011, 79-80.

27. Sugli onori accordati a Timostene (II) e rinnovati a un suo discendente (Timostene III), vd. OSBORNE 1981, 112-113 (D 43), 196-198 (D 90); OSBORNE 1982, 116-117 (comm. a D 43), 179-180 (comm. a D 90).

28. Per quanto precede, vd. specialmente SCAFURO 2009.

29. Limitatissima e parziale, me ne rendo ben conto: fra l'altro, non sono stati presi in

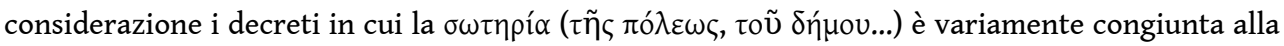

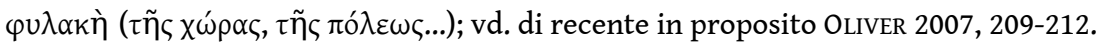

30. Cfr. CARTLEDGe 1987, 143-144. 
31. FLOWER 1988. Osservo qui incidentalmente che non tutte le affermazioni contenute in questo importante contributo sono persuasive.

32. Vd. al riguardo fra gli altri KATSAROS 2007, 221-225; VALERIO 2013, 83-94.

33. In senso nettamente contrario si esprime GoUKOWSKY 2016, lxxx-xciii.

34. MuCCIOLI 1997, 121-122. Vd. inoltre MucCIOLI 1999, 361 con nota 956.

35. E l'associazione avviene attraverso un giro di parole non propriamente scontato. Com'è ampiamente noto, Diodoro (XI 26,5-6) riferisce che, dopo Imera, Gelone sarebbe stato acclamato dai partecipanti all'assemblea come euergetes, soter e basileus: la testimonianza diodorea può apparire sospetta e viziata da un linguaggio anacronistico, ma più di un elemento induce quanto meno a sfumare il giudizio (vd. di recente MORGAN 2015, 82 con bibliografia; inoltre MITCHELL 2013, 85 nota 68 ).

36. Mi concentrerò qui di seguito sul valore teorico-politico di soteria in Platone (e i suoi scolari), ma quel concetto ha nei Dialoghi un'estensione decisamente più vasta: così, la Repubblica ci permette di constatare come soteria e sozo stiano certo a significare la salvezza della kallipolis (465 $\mathrm{d} 7-8$ ), ma anche una salvezza che riguarda la sorte dell'anima dopo la morte (621 c 1 [cfr. Fedone $107 \mathrm{c}-\mathrm{d}]) ;$ pregevole, in proposito, MENN 2013.

37. Una simile via d'accesso alla Philosophenherrschaft - di cui si fa parola prima a 473 c 11-e 4, poi a 499 b 1-c 5 - sembra indicata da Platone anche in un celebre e discusso passo delle Leggi (708 e 1-712 a 7): cfr. TRAMPEDACH 1994, 209-215 e soprattutto VEGETTI 2005, 162-168, ove riferimenti; propende invece per un ripensamento, nell'ultima opera platonica, di questo paradigma della Repubblica S CHOFIELD 1999, 28-45, partic. 38-44 con le note corrispondenti; ulteriore, ampia, bibliografia sul passo delle Leggi in PRAUSCELLO 2014, 76-77.

38. Vd. SANDVoss 1971; inoltre per esempio PAQUET 1973, 260 con nota 3.

39. Su 960 c 8-d 1 rinvio al commento di SCHÖPSDAU 2011, 586-588.

40. Per qualche cenno su questa categoria di decreti, di cui abbiamo considerato in precedenza la prima attestazione, vd. CAMASSA 2016, 266-267.

41. HAYES 2015, 76.

42. Non posso qui trattare in genere la questione, estremamente dibattuta, dei rapporti fra elaborazione teorica e impegno politico concreto in seno all'Accademia - e a partire da essa: equilibrata mi sembra per esempio la posizione di KLOSKO 2006, 199-200. E vd. ora HAAKE 2020, partic. 73-74 con ampia messe di riferimenti bibliografici.

43. SLATER 1988 (con la bibliografia precedente).

44. Per ciò che segue, vd. CAMASSA 2018.

\section{RIASSUNTI}

Soter e soteria, che da soter deriva, non corrispondono sempre ai nostri «salvatore» e «salvezza». Questa resa ci permette tuttavia di catturare un valore semantico profondo e rivelatore che inerisce, nel corso del tempo, ai due vocaboli. Occorre tentare allora una periodizzazione: da quando soter e soteria divengono vocaboli di bruciante attualità nel panorama della cultura greca e non investono più solo il piano divino, del soccorso richiesto ai numi (salvatori) in circostanze difficili, ma anche il piano umano, dell'atto di fiducia incondizionatamente riposta in un individuo eccezionale dinanzi a una crisi senza apparente via di uscita? Siamo inconsapevolmente portati a ritenere che soter, e soteria insieme con soter, costituiscano le cifre 
peculiari dell'età moderna della storia greca - dell'ellenismo - , un portato della sua trasformazione in senso carismatico. Ma a guardar meglio questa armatura fattuale e ideologica, che si dispiegherà appieno dopo Alessandro, è ben presente nella realtà del mondo ellenico almeno dalla seconda metà del V secolo. Se non già dai decenni, o dalla rivisitazione dei decenni, in cui incombeva la minaccia persiana e i Greci hanno ottenuto quasi miracolosamente la salvezza contro un nemico così forte da schiacciarli. A segnare una vera cesura sul piano qualitativo e quantitativo sono comunque gli sviluppi che si producono con la lotta cruciale per l'egemonia sull'Ellade. È allora che gli antichi equilibri si spezzano e la drammaticità delle situazioni da affrontare si fa manifesta; solo la fuoriuscita dai quadri istituzionali e mentali consueti, se del caso mediante agenti umani straordinari - il ricorso a un soter -, sembra una soluzione capace di porre rimedio al malessere che attanaglia la polis - rimedio coincidente in ultima analisi con la soteria.

Soter and soteria, deriving from soter, do not always correspond to "saviour" and "salvation". This particular rendering nevertheless enables a deeper, more telling semantic component to be grasped which, over the course of time, became inherent to the two lexical items. Establishing a time frame is therefore necessary: when did soter and soteria become lexical items of acute relevance in Greek culture, no longer pertaining only to the realm of the divine when seeking help from the (saving) gods in adverse circumstances, but also to humanity in the form of the unconditional trust placed in an exceptional individual to deal with a seemingly intractable crisis? Unconsciously one may be led to believe that soter, and soteria with soter, represent the salient characteristics of the modern era of Greek history - Hellenism - and are harbingers of its transformation in a charismatic sense. But further reflection shows that this factual and ideological scaffolding, which peaked immediately in the wake of Alexander, was already present in the Hellenic world at least as far back as the second half of the $5^{\text {th }}$ century B.C., if not even in the decades (or the re-examination of those decades) during which Persia loomed menacingly and the Greeks were almost miraculously saved from an enemy capable of annihilating them. A real turning point, in both qualitative and quantitative terms, was encapsulated in the progress achieved through the crucial struggle for hegemony over Hellas. It was then that long-standing equilibriums were overturned and the critical nature of the various situations that had to be dealt with became evident; only by drawing back from the customary institutional and mental frameworks, where possible with the aid of extraordinary human agents (recourse to a soter), could a solution be found capable of healing the malaise gripping the polis - a remedy that in the final analysis coincides with soteria.

\title{
INDICE
}

Keywords : Greek History, Soter, Soteria, Saviour, Salvation

Parole chiave : Storia greca, Soter, soteria, Salvatore, salvezza

\author{
AUTORE \\ GIORGIO CAMASSA \\ già ordinario di Storia greca presso l'Università di Udine \\ giorgiocamassa51(at)gmail.com
}

